\title{
Strong-LAMP Assay Based on a Strongyloides spp.-Derived Partial Sequence in the $18 S$ rRNA as Potential Biomarker for Strongyloidiasis Diagnosis in Human Urine Samples
}

\author{
Pedro Fernández-Soto $\mathbb{D}^{1},{ }^{1}$ Carmen T. Celis-Giraldo $\mathbb{D}^{2}{ }^{2}$ Coralina Collar-Fernández, ${ }^{1}$ \\ Óscar Gorgojo $\mathbb{D}^{1},{ }^{1}$ Milena Camargo $\mathbb{D}^{3,4}{ }^{3,4}$ José Muñoz, $^{5}$ Joaquín Salas-Coronas, ${ }^{6}$ \\ Manuel A. Patarroyo $\mathbb{D}^{3,7}$ and Antonio Muro ${ }^{1}{ }^{1}$ \\ ${ }^{1}$ Infectious and Tropical Diseases Research Group (e-INTRO), Biomedical Research Institute of Salamanca-Research Centre for \\ Tropical Diseases at the University of Salamanca (IBSAL-CIETUS), Faculty of Pharmacy, University of Salamanca, \\ Salamanca 37007, Spain \\ ${ }^{2}$ Animal Science Faculty, Universidad de Ciencias Aplicadas y Ambientales (U.D.C.A), Bogotá 111166, Colombia \\ ${ }^{3}$ Molecular Biology and Immunology Department, Fundación Instituto de Inmunología de Colombia (FIDIC), \\ Bogotá 111321, Colombia \\ ${ }^{4}$ PhD Programme in Biomedical and Biological Sciences, School of Medicine and Health Sciences, Universidad del Rosario, \\ Bogotá 112111, Colombia \\ ${ }^{5}$ ISGlobal, Barcelona Ctr. Int. Health Res. (CRESIB), Hospital Clinic-Universitat de Barcelona, Barcelona 08036, Spain \\ ${ }^{6}$ Unidad de Medicina Tropical, Hospital de Poniente, El Ejido 04700, Almería, Spain \\ ${ }^{7}$ Basic Sciences Department, School of Medicine and Health Sciences, Universidad del Rosario, Bogotá 112111, Colombia
}

Correspondence should be addressed to Manuel A. Patarroyo; mapatarr.fidic@gmail.com and Antonio Muro; ama@usal.es

Received 28 August 2019; Accepted 24 April 2020; Published 31 May 2020

Academic Editor: Lucio Castellano

Copyright (C) 2020 Pedro Fernández-Soto et al. This is an open access article distributed under the Creative Commons Attribution License, which permits unrestricted use, distribution, and reproduction in any medium, provided the original work is properly cited.

\begin{abstract}
Human strongyloidiasis a soil-transmitted infection caused by Strongyloides stercoralis is one of the most neglected amongst the so-called Neglected Tropical Diseases (NTDs). S. stercoralis is a nematode, which is distributed worldwide; it has been estimated that it could affect millions of people, mainly in tropical and subtropical endemic regions. The difficulties of diagnosis lead to infection rates being underreported. Asymptomatic patients have chronic infections that can lead to severe hyperinfection syndrome or disseminated strongyloidiasis in immunocompromised patients. Strongyloidiasis can easily be misdiagnosed because conventional faecal-based techniques lack of sensitivity for the morphological identification of infective larvae in faeces. None of the currently used molecular methods have used urine samples as an alternative to faecal samples for diagnosing strongyloidiasis. This study was thus aimed at comparing, for the first time, the use of a new loop-mediated isothermal amplification (LAMP) molecular assay (Strong-LAMP) to traditional methods on patients' urine samples. Twenty-four urine samples were taken from patients included in a study involving two Spanish hospitals for strongyloidiasis screening using parasitological and serological tests. Strongyloides larvae were found in 11 patients' faecal samples, thereby ascertaining that they had the disease. Other patients had high antibody titres but no larvae were found in their faeces. All urine samples were analysed by PCR and Strong-LAMP assay. No amplification occurred when using PCR. Strong-LAMP led to detecting S. stercoralis DNA in urine samples from patients having previously confirmed strongyloidiasis by parasitological tests and/or a suspicion of being infected by serological ones. The Strong-LAMP assay is a useful molecular tool for research regarding strongyloidiasis in human urine samples. After further validation, the Strong-LAMP assay could also be used for complementary and effective diagnosis of strongyloidiasis in a clinical setting.
\end{abstract}




\section{Introduction}

Strongyloidiasis is an infection caused by the parasitic nematodes from the genus Strongyloides: S. stercoralis and to a lesser extent Strongyloides fuelleborni. Originally known as "anguilulosis" or "Cochinchina diarrhoea", the World Health Organisation (WHO) now considers it a neglected tropical disease (NTD) [1,2]. S. stercoralis has a cosmopolitan distribution in tropical and subtropical regions [3]. It can also be found in temperate areas, such as the Mediterranean region, southern USA, and Japan. Regarding S. fuelleborni, although primarily affecting nonhuman primates, human cases have also been described in Africa and Southeast Asia, mainly in Papua New Guinea $[4,5]$.

Strongyloidiasis worldwide has currently been calculated as ranging from 30-100 million infected people, mainly in low-income countries and those having poor sanitary conditions $[6,7]$. Such variation regarding its estimation is largely due to its asymptomatic clinical picture, the tremendous difficulties regarding its diagnosis, and the affected people's lack of access to a health system. The disease's prevalence is considered to be greatly underestimated [8]. S. stercoralis is an autochthonous parasite in Spain all along its Mediterranean coastline, particularly in La Safor region within the province of Valencia, Spain, where it reaches $12.4 \%$ in high-risk groups related to agricultural work $[6,9]$; cases have also been reported on the banks of the Ebro river [10]. Most European cases have been concerned with parasitosis imported by immigrants from strongyloidiasis-endemic areas, to a lesser extent, cases of travellers visiting such areas $[11,12]$.

Strongyloidiasis clinical manifestations depend on parasite development and invasion stage, its self-infection capability, and a patient's immunological state. This may appear as an acute infection and chronic infection and produce a hyperinfection syndrome and/or a disseminated infection. Acute strongyloidiasis is not common and usually appears in travellers returning from a highly-endemic area suffering from pruritic dermatitis (due to the larvae penetrating the skin), pneumonitis accompanied by cough and expectoration (when the larvae enter the lungs), and fever. The parasites produce gastrointestinal pain accompanied by diarrhoea, nausea, and, occasionally, vomiting when they reach the intestines. Chronic (or low intensity) strongyloidiasis is usually asymptomatic, although it can have slight to moderate symptomatology, accompanied by gastrointestinal, pulmonary and cutaneous manifestations, and eosinophilia (in $75 \%$ of patients) [13].

It can produce the hyperinfection syndrome in immunosuppressed individuals when the larvae migrate, accompanied by more severe intestinal and pulmonary manifestations, fever, weakness, and a greater amount of larvae in faeces and sputum. Immunosuppressive treatments involving corticosteroids, solid or haematopoietic organ transplants, cancer, and HTLV-1 infection are considered the most important associated risk factors [4], along with malnutrition and associated infections in areas having high endemicity [14]. Anti-TNF therapies (stand alone or in combination with glucocorticoids) have favoured the development of clinical pictures and hyperinfections as they affect Th2 cells' immune response $[15,16]$.

The larvae can cross the blood-brain barrier, producing encephalitis and up to $87 \%$ mortality rates. The treatment usually used for strongyloidiasis is no longer effective at this point [17]; screening individuals suspected of having strongyloidiasis before immunosuppressive treatment is thus essential [4]. Ivermectin has been seen to be the most therapeutically effective drug used in control strategy; it continues being the drug of first choice regarding other options such as albendazole, thiabendazole, or mebendazole which are less effective and less safe $[6,16,18,19]$.

However, diagnosis is undoubtedly the main problem regarding strongyloidiasis due to little knowledge being available concerning the disease, its effects in nonendemic areas, current diagnostic techniques having little sensitivity and specificity, the parasitological methods requiring specialised personnel, and centres and no gold standard for diagnosis. This means that the case definition and the possible validation of new diagnostic methods are enormously hampered [20].

Current parasitological and immunological S. stercoralis diagnostic methods are thus being complemented by molecular methods $[17,21,22]$. Different approaches to the molecular detection of $S$. stercoralis in faecal samples have been developed from the description of the Strongyloides spp. $18 \mathrm{~S}$ ribosomal subunit sequence, using polymerase chain reaction (PCR), both simple and nested techniques, and real time-PCR (RT-PCR) [8, 23]. Another recent molecular alternative method for diagnosing strongyloidiasis in patients' faecal samples [24] is the loop-mediated isothermal amplification (LAMP) of nucleic acids which has numerous advantages over other more complex molecular diagnosis techniques $[25,26]$.

LAMP is currently considered a technique having great potential for use in field conditions, mainly in endemic areas, as a future, highly effective, point-of-care testing method [27]. Fernández-Soto and colleagues [28] have developed a new LAMP method called Strong-LAMP for the molecular detection of Strongyloides spp. in urine and faecal samples in a murine model. It has also been used for analysing stool samples from patients previously diagnosed by parasitological and molecular (RT-PCR) methods, thereby making it a highly efficient diagnosis technique [28]. In this work, the Strong-LAMP is used for the first time in human urine samples from patients attending at two hospitals in Spain and compared with parasitological and serological methods.

\section{Materials and Methods}

2.1. Obtaining the Samples. The urine samples used in this study were obtained from patients (mostly immigrants) attending the Tropical Medicine Unit in Hospital Clínic de Barcelona (Barcelona, Spain) and Hospital de Poniente (El Ejido Almería, Spain) as part of a strongyloidiasis diagnosis screening study. The inclusion criteria were being a patient having simultaneous blood, faeces, and urine samples; having a positive parasitological and/or serological diagnosis for Strongyloides who had been recruited for a 
TABLE 1: Nucleotide sequences from a set of primers selected from the 329 bp sequence (GenBank Acc. num.: AJ417026.1) for LAMP amplification of Strongyloides spp. DNA [28].

\begin{tabular}{lcc}
\hline Primer & Length $(\mathrm{bp})$ & Sequence $\left(5^{\prime}-3^{\prime}\right)$ \\
\hline F3 & 21 & ACACGCTTTTTATACCACATT \\
B3 & 18 & GTGGAGCCGTTTATCAGG \\
FIP & 49 & ACCAGATACACATACGGTATGTTTTGGATTTGATGAAACCATTTTTTCG \\
BIP & 43 & ATCAACTTTCGATGGTAGGGTATTGCCTATCCGGAGTCGAACC \\
\hline
\end{tabular}

multicentre study for strongyloidiasis diagnosis; a convenience sampling was thus performed, considering the abovementioned criteria. Each individual's clinical picture was recorded. All the patients included in the study were first analysed by S. stercoralis serology using a Microwell ELISA kit (IVD Research, Inc., Carlsbad, CA) (https://ivdresearch.com/elisa/strongyloidesserum-antibody-detection-microwell-elisa/) and coproparasitological analysis performed on only one sample (Ritchie technique and agar-plate culture for patients attending the Hospital de Poniente and just agar-plate culture for the Hospital Clínic de Barcelona patients) for detecting S. stercoralis. Samples whose optical density (OD) values were $\geq 1$ in the IVD-ELISA test were considered positive (according to the manufacturers' specifications). The study involved 24 urine samples: 16 from Hospital Clínic (Barcelona) and 8 from Hospital de Poniente (Almería). All samples were sent to CIETUS in $10 \mathrm{~mL}$ tubes and stored frozen until use.

2.2. Ethical Considerations. Both hospitals' Ethics Committees approved the study; the urine samples were obtained after the patients had signed informed consent forms regarding their analysis.

\subsection{Obtaining and Preparing the DNA Samples}

2.3.1. Obtaining and Preparing Strongyloides venezuelensis DNA. DNA from $S$. venezuelensis infective filiform larvae (L3) was used as amplification positive control for PCR and LAMP reactions; their biological cycle is routinely maintained in experimentally-infected Wistar rats in the CIETUS, Universidad de Salamanca. A NucleoSpin Tissue kit (Machery-Nagel) was used for extracting DNA from the larvae, following the manufacturers' instructions. The DNA concentration was measured on a NanoDrop spectrophotometer (ND-1000) and adjusted to the final $5 \mathrm{ng} / \mu \mathrm{L}$ concentration. This DNA $(2 \mu \mathrm{L})$ was then used as positive control for all subsequent PCR and LAMP reactions.

\subsubsection{Obtaining and Preparing DNA from Patients' Urine.} This involved taking $2 \mathrm{~mL}$ aliquots of patients' urine from the unfrozen vials; the rest remained frozen at $-20^{\circ} \mathrm{C}$. The vials to be analysed were spun at $4,000 \mathrm{rpm}$ for $15 \mathrm{~min}$ to obtain sediment for extracting the DNA using the i-genomic Urine DNA Extraction Mini Kit (Intron Biotechnology), following the manufacturers' instructions. A NanoDrop spectrophotometer (ND-1000) was used for measuring the DNA concentration from each urine sample $(100 \mu \mathrm{L}$ elution volume); they were then labelled and stored until use at $-20^{\circ} \mathrm{C}$ in two vials ( $50 \mu \mathrm{L}$ in each).
2.4. Amplification Target and Specific Primers for LAMP Amplifying Strongyloides spp. DNA. The Strong-LAMP method previously validated and developed at CIETUS was used for Strongyloides spp. DNA amplification [17]. Briefly, the selected amplification target was a Strongyloides venezuelensis partial 18S rRNA gene (GenBank Accession number: AJ417026.1) 329 base pair (bp) sequence; Primer Explorer V.4 software (https://primerexplorer.jp/e/) was used for designing a set of four primers (F3, B3, FIP, and BIP) on this sequence (Table 1 shows the selected primer sequences). A Fisher Scientific synthesis kit was used, and purified products were suspended in ultrapure water at $100 \mathrm{pmol} / \mu \mathrm{L}$ final concentration.

\subsection{Molecular Analysis of Patients' Urine Samples}

2.5.1. PCR Analysis of F3 and B3 External Primers. The urine samples were analysed by PCR using F3 and B3 external primers from the set of 4 primers for the LAMP assay; correct PCR functioning had already been verified with $S$. venezuelensis DNA. A touchdown PCR (TD-PCR) was carried out consisting of decreasing the annealing temperature by one degree per each amplification cycle for guaranteeing a suitable range of annealing temperatures for correct target sequence amplification [29]; briefly, TD-PCR was performed as follows: $94^{\circ} \mathrm{C}$ for 60 seconds and a touchdown program involving 18 cycles; Table 2 shows the reaction mixture and amplification conditions used in the TD-PCR. A 96-well thermal cycler (Gradient Mastercycler, Eppendorf) was used for all PCR reactions.

2.5.2. LAMP Analysis of Patients' Urine Samples. All the urine samples were analysed by the Strong-LAMP previously described by Fernández-Soto et al. [28]. Table 3 describes the reaction mixture used. All reactions were incubated for $60 \mathrm{~min}$ at $63^{\circ} \mathrm{C}$ in a heating block (K Dry-Bath) plus $10 \mathrm{~min}$ at $80^{\circ} \mathrm{C}$ for deactivating the enzyme and stopping the reaction; $2 \mu \mathrm{L}$ DNA from each urine sample were used for amplification; S. venezuelensis DNA $(2 \mu \mathrm{L})$ and ultrapure water instead of DNA $(2 \mu \mathrm{L})$ were used as positive and negative controls, respectively.

\subsubsection{Detecting the Amplification Products}

(1) PCR. PCR amplification products were detected on $1.5 \%$ agarose gels ( $100 \mathrm{~mL} 0.5 \mathrm{X}$ TBE, $1.5 \mathrm{~g}$ agarose), stained with ethidium bromide at $60 \mathrm{~V}$ for $20 \mathrm{~min}$ and then at $90-100 \mathrm{~V}$ for one hour. The gels were visualised and photographed using an ultraviolet imaging system (UVITEC Gel Documentation System, Cambridge, UK). 
TABLE 2: Reaction mixture (a) and amplification conditions used in TD-PCR using F3 and B3 external primers (b). Taq polymerase, buffer, $\mathrm{MgCl}_{2}$, and dNTPs were supplied by Intron.

(a)

\begin{tabular}{lc}
\hline Components & Volume $(\mu \mathrm{L})$ \\
\hline $\mathrm{H}_{2} \mathrm{O}$ & 17.1 \\
10x buffer & 2.5 \\
$\mathrm{MgCl} 2(25 \mathrm{mM})$ & 1.5 \\
$\mathrm{dNTPs}(2.5 \mathrm{mM})$ & 0.5 \\
$\mathrm{~F} 3($ pmol $)$ & 0.5 \\
B3 (pmol) & 0.5 \\
Taq polymerase $(2 \mathrm{U})$ & 0.4 \\
Template DNA & 2 \\
Total & 25 \\
\hline
\end{tabular}

(b)

\begin{tabular}{lcc}
\hline Temperature $\left({ }^{\circ} \mathrm{C}\right)$ & Time $(\mathrm{sec})$ & Cycle \\
\hline 94 & 60 & $\mathrm{X} 1$ \\
94 & 20 & \\
$57-52$ & 20 & $\mathrm{X} 2$ \\
72 & 30 & \\
94 & 60 & \\
51 & 20 & $\mathrm{X} 15$ \\
72 & 30 & \\
72 & 60 & $\mathrm{X} 1$ \\
\hline
\end{tabular}

TABle 3: Reaction mixture used in Strong-LAMP assays. Bst polymerase, buffer, and $\mathrm{MgSO}_{4}$ were supplied by New England Biolabs, betaine by SIGMA, and the dNTPs by Intron.

\begin{tabular}{lc}
\hline Component & Volume $(\mu \mathrm{L})$ \\
\hline $\mathrm{H}_{2} \mathrm{O}$ & 7.7 \\
Betaine $(1 \mathrm{M})$ & 5 \\
$\mathrm{MgSO}_{4}$ & 1.5 \\
$\mathrm{dNTPs}(2.5 \mathrm{mM})$ & 3.5 \\
10x buffer & 2.5 \\
$\mathrm{FIP}(40 \mathrm{pmol} / \mu \mathrm{L})$ & 0.4 \\
$\mathrm{BIP}(40 \mathrm{pmol} / \mu \mathrm{L})$ & 0.4 \\
$\mathrm{~F} 3(5 \mathrm{pmol} / \mu \mathrm{L})$ & 0.5 \\
$\mathrm{~B} 3(5 \mathrm{pmol} / \mu \mathrm{L})$ & 0.5 \\
Bst polymerase 2.0 & 1 \\
DNA & 2 \\
Total & 25 \\
\hline
\end{tabular}

(2) Strong-LAMP. LAMP amplification products were visually detected by observing white turbidity at the bottom of the reaction tube and by colorimetric change on adding $2 \mu \mathrm{L}$ SYBR Green I fluorescent dye (Invitrogen) (1:10; $10,000 \mathrm{X})$ to each reaction tube. Green indicated a positive result and orange a negative one (i.e., maintaining the dye's original colour). Colorimetric results were verified by $1.5 \%$ agarose electrophoresis for observing the characteristic pattern of bands which appears in positive LAMP results. The gels were then photographed and the images saved in digital format for editing.

2.6. Statistical Analysis. Stata MP 14.0 statistical software was used for analysing the data. A descriptive analysis was made; quantitative variables are shown as Medians or Means with their corresponding dispersion measures (Interquartile range-IQR or Standard Deviation-SD); 5\% significance confidence intervals were calculated for each test. Agreement between serological and coproparasitological screening tests with Strong-LAMP results in this study was quantified and analysed using the kappa coefficient $(\kappa)$, interpreted as: $<0.00$ poor, $0 \leq \kappa \leq 0.2$ slight, $0.21 \leq \kappa \leq 0.40$ fair, $0.41 \leq \kappa$ $\leq 0.60$ moderate, $0.61 \leq \kappa \leq 0.80$ substantial, and $>0.80$ almost perfect [30].

\section{Results}

3.1. Serological and Parasitological Data. The study results showed that $54 \%$ of the samples came from Latin-American patients (mainly from Bolivia (9/24) and the remainder from Africa (specifically Gambia (4/24) and Guinea-Bissau $(2 / 24))$. The patients' epidemiological data stated that $62.5 \%(15 / 24)$ were male, most being aged from 30 to 60 years old $(75 \%)$, with a mean $=41.1(\mathrm{SD}=12.6)$. Regarding their clinical pictures, 50\% (12/24) of the target population had symptoms such as abdominal pain, diarrhoea, urticaria, and pruritus. Eosinophilia was observed in 45.8\% (11/24) of the population, with a median $=870(\mathrm{IQR}=680-1900)$, accompanied by high IgE levels in $52.15 \%$ of them (12/23), with a median $=1320.5(\mathrm{IQR}=542-2438)$ (Table 4$)$. Strongyloidiasis diagnosis test revealed that $87.5 \%$ of the study population had $\geq 1$ titres for IVD-ELISA with a median $=$ $2.22(\mathrm{IQR}=1.71-4.52)$. However, 37.5\% (0.19-0.614 95\% CI) of the population was positive by coproparasitological analysis by agar culture plate method (individual results in Supplementary material Tables 1 and 2. Once diagnosed, $95.8 \%(23 / 24)$ of the individuals were treated with ivermectin; all but one patient were followed-up.

3.2. Human Urine Samples Analysed by TD-PCR F3-B3 Assay. Figure 1 shows the TD-PCR amplification results; there was no amplification in any of the samples analysed.

3.3. Strong-LAMP Analysis of Patients' Urine Samples. It was found that $50 \%$ of the individuals $(0.25-0.6795 \% \mathrm{CI})$ were positive according to test parameters. Figure 2 shows Strong-LAMP results from analysing the patients' urine samples from the Hospital Clínic de Barcelona and Figure 3 from the Hospital de Poniente; results from other tests are also shown.

3.4. Agreement between Screening Analysis and StrongLAMP. Strong-LAMP results were compared to the IVD test results and then to the coproparasitological analysis results. The numbers in the figure represent the total of samples proving positive in each test analysed. The scale on the lower axis indicates the percentage in relation to the total of 
TABle 4: Descriptive statistics regarding the population's epidemiological characteristic, clinical status (a), and haematological results (b).

(a)

\begin{tabular}{lc}
\hline Epidemiological characteristic & Total $(n(\%))$ \\
\hline Origin & \\
Bolivia & $9(37.5)$ \\
Gambia & $4(16.6)$ \\
Ecuador & $2(8.3)$ \\
Guinea Bissau & $2(8.3)$ \\
Spain & $2(8.3)$ \\
Other & $5(20.83)$ \\
Gender & \\
Female & $9(37.5)$ \\
Male & $15(62.5)$ \\
Age (years) & \\
$<30$ & $4(16.6)$ \\
$30-60$ & $18(75)$ \\
$>60$ & $2(8.3)$ \\
Clinical status & \\
Asymptomatic & $12(50)$ \\
Symptomatic & $12(50)$ \\
\hline
\end{tabular}

(b)

\begin{tabular}{llcc}
\hline Laboratory test & $n$ & Median & IQR \\
\hline Haematological result & & & \\
Eosinophils & 23 & 400 & $(100-870)$ \\
IgE & 22 & 358 & $(72-1653)$ \\
\hline
\end{tabular}

${ }^{1}$ Only one person by country (Colombia, Cuba, Mali, Nigeria, and Portugal). ${ }^{2}$ Optical density (OD) according to the maker's specifications.

positive individuals (Figure 4); 37.5\% agreement was observed and poor correlation according to $\kappa$ value for IVD and Strong-LAMP analysis $(\kappa=-0.25 ; p=0.968)$. Such data differed from that observed regarding coprology and Strong-LAMP results where agreement was $79.17 \%$. The $\kappa$ value gave moderate correlation $(\kappa=0.5833 ; p=0.0016)$, according to the classification described above.

\section{Discussion}

One of the main problems regarding strongyloidiasis continues being the lack of a true gold standard for diagnosing the disease. Even though parasitological methods continue being considered "clinical or certainty diagnosis" (i.e., they enable visualising the larvae in faecal samples), they involve great disadvantages, mainly regarding their sensitivity $[6,21]$.

Serological methods also have problems regarding sensitivity (especially during the disease's acute phase) and specificity due to often producing crossed reactions concerning a diagnosis of other nematodes. PCR-based molecular methods and variants (e.g., RT-PCR) have been advanced during recent years as the most suitable ones for diagnosing strongyloidiasis, even though requiring complex infrastructure and specialised equipment and personnel which are not available in routine practice and are expensive and very difficult to perform in field conditions in strongyloidiasisendemic areas $[17,31]$.

The lack of a suitable and standardised diagnosis method has led to underestimating the disease's real prevalence. Although migrants were screened in this study, it was interesting that most individuals came from Latin America, mainly from Bolivia. Studies have been inconsistent when reporting the disease's prevalence in Latin America; values have been higher than 20\% in Argentina, Ecuador, Venezuela, Perú, and Brazil [32] whilst a study by Gétaz [33] in Bolivia estimated prevalence at $20.0 \%$. Eosinophilia being found in $45.8 \%(11 / 24)$ of the target population accompanied by increased $\operatorname{IgE}$ production in 9 patients is parameters which have been reported in strongyloidiasis cases [34]. However, individuals suffering eosinophilia cannot always be identified as this depends on the parasite's cycle and concomitant infections [35]. Regarding IgE levels, normal levels have been reported in individuals infected by Strongyloides stercoralis who also proved positive for human T-cell lymphotropic virus type 1 (HTLV-1) [36].

Difficulties regarding diagnosis are especially important regarding immunocompromised people or immunosuppression candidates (e.g., in transplants) who could develop hyperinfection leading to a fatal outcome for the patient [4]. A study by Luvira et al. [37], involving patients who were immunocompromised due to different causes, analysed factors such as age, gender, and level of immunocompromise and reported that they were not associated with strongyloidiasis; prevalence ranged from 5\%-7\% and coproparasitological analysis in agar was the most sensitive technique in this study [37]. New sensitive, specific, technically easy, and economically affordable diagnosis methods must thus be developed; they must also become incorporated into routine clinical practice as a screening method for strongyloidiasis in patients who are candidates for immunosuppressive treatment.

However, most molecular diagnosis methods for detecting S. stercoralis have been based on using faecal or soil samples in the search for larvae. Faecal samples involve problems associated with collecting (serial, at least 3 samples), processing (usually requiring them to be concentrated), and storing them. Urine analysis is an alternative for making a diagnosis $[20,28,38,39]$. A new molecular diagnosis method such as LAMP for use on urine samples thus provides an interesting approach, combining this technique's advantages over other molecular methods (i.e., PCR) and the advantages of analysing urine samples. Previous studies have evaluated the parasite's presence in urine; a study by Lodh et al. [40] detected $S$. stercoralis-derived DNA by molecular methods, Formenti et al. [41] evaluated parasite diagnosis by conventional and molecular methods such as q-PCR analysing urine samples and faecal material; they found that qPCR urine analysis sensitivity was just $17 \%$ compared to faecal material analysis having 63\% sensitivity [41].

In a previous work, Strong-LAMP adaption of the LAMP method for detecting Strongyloides venezuelensis has proved 


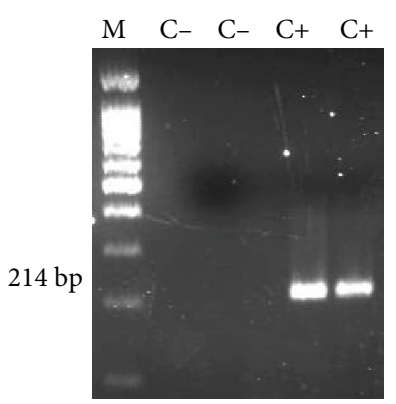

(a)

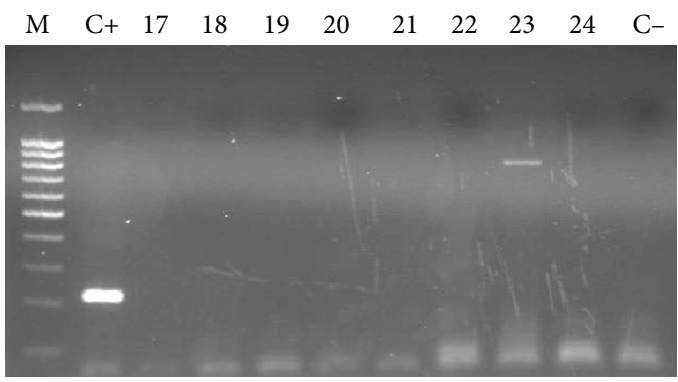

(b)

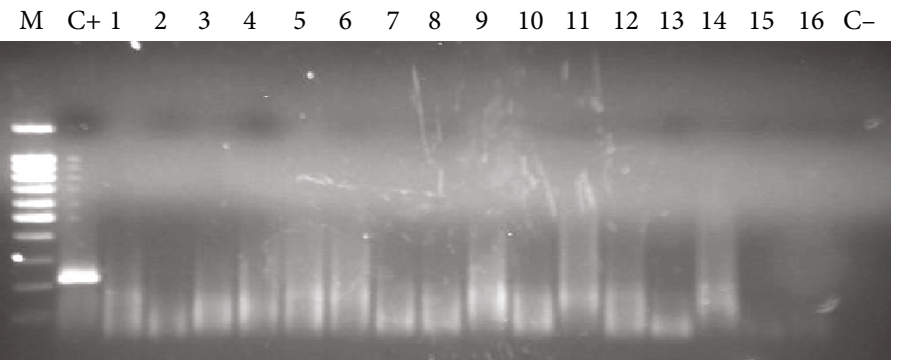

(c)

FIGURE 1: TD-PCR analysis of patients' urine samples. (a) PCR for verifying F3 and B3 primer functioning for amplifying the 214 bp fragment. (b) Samples from the Hospital de Poniente (El Ejido, Almería). (c) Samples from the Hospital Clínic de Barcelona. M: molecular weight marker (DNA Ladder 100 bp PLUS BLUE); C+: Strongyloides venezuelensis DNA (positive control); C-: ultrapure water, no DNA (negative control).

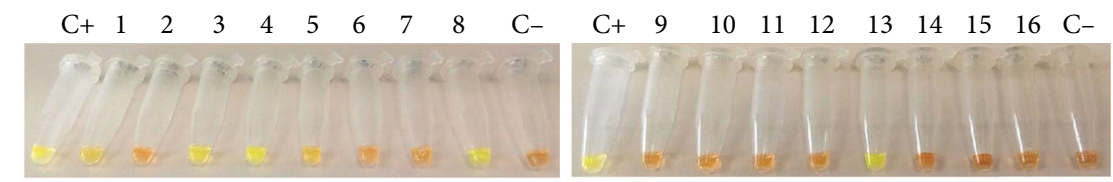

M

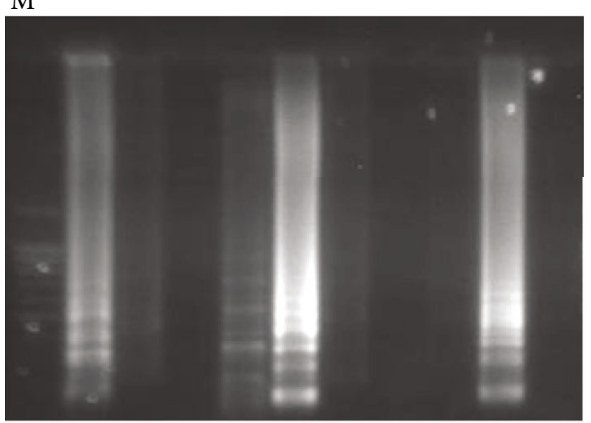

(a)

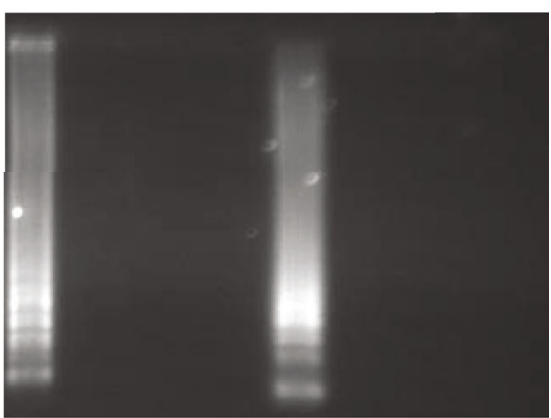

(b)

\begin{tabular}{|c|c|c|c|c|c|c|c|c|c|c|c|c|c|c|c|c|c|c|c|c|}
\hline Technique/sample & $\mathrm{C}+$ & 1 & 2 & 3 & 4 & 5 & 6 & 7 & 8 & C- & $\mathrm{C}+$ & 9 & 10 & 11 & 12 & 13 & 14 & 15 & 16 & C- \\
\hline & & 4.4 & 1.5 & 13.7 & 2.2 & 14 & 1.5 & 1.2 & 4 & & & 2 & 1.7 & 7.2 & 2.1 & 0 & 2 & 1.2 & 11.9 & \\
\hline Agar culture plate method & & + & - & - & - & + & - & _ & + & & & - & _- & - & - & + & - & - & - & \\
\hline
\end{tabular}

(c)

Figure 2: Strong-LAMP analysis of urine samples from patients attending Hospital Clínic de Barcelona (Barcelona). (a) Colorimetric results from adding SYBR Green I. (b) Agarose gel electrophoresis results. (c) Serological (IVD index, OD values) and agar plate culture results. M: molecular weight marker (DNA Ladder 100 bp PLUS BLUE); C+: Strongyloides venezuelensis DNA (positive control); C-: ultrapure water, no DNA (negative control). 

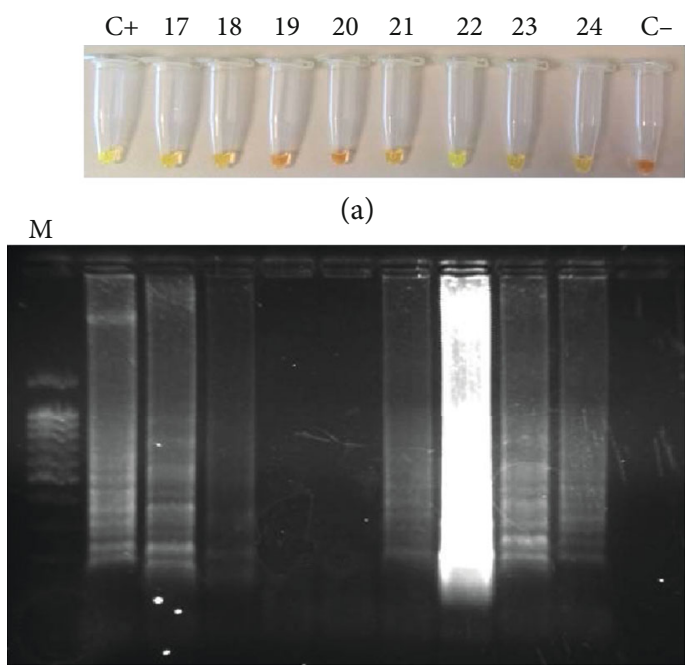

(b)

\begin{tabular}{lcccccccccc}
\hline Technique/sample & $\mathrm{C}+$ & 17 & 18 & 19 & 20 & 21 & 22 & 23 & 24 & $\mathrm{C}-$ \\
\hline Serology (IVD) & & 4.52 & 4.10 & 1.39 & 1.97 & 0.48 & 3.22 & 8.31 & 0.39 & \\
Ritchie technique & & + & + & - & - & + & + & - & + & \\
$\begin{array}{l}\text { Agar culture plate } \\
\text { method }\end{array}$ & & + & + & + & - & - & + & + & - & \\
\hline
\end{tabular}

(c)

Figure 3: Strong-LAMP analysis of urine samples from patients attending Hospital de Poniente (El Ejido, Almería). (a) Colorimetric results by adding SYBR Green I. (b) Agarose gel electrophoresis results. (c) Serological (IVD index, OD values), Ritchie technique and agar plate culture results. M: molecular weight marker (DNA Ladder 100 bp PLUS BLUE); C+: Strongyloides venezuelensis DNA (positive control); C-: ultrapure water, no DNA (negative control).

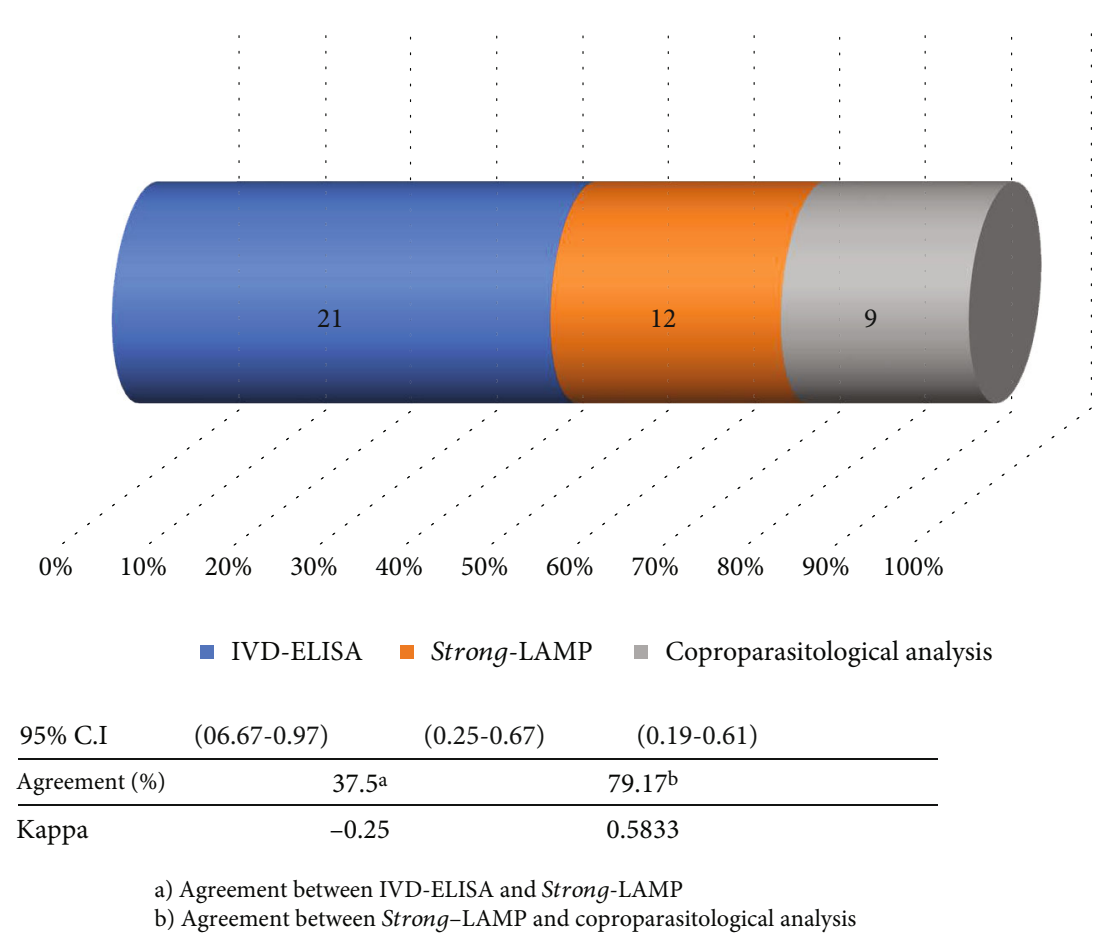

FIgURe 4: Agreement between Strong-LAMP, IVD detection techniques, and coproparasitological analysis in the studied samples with strongyloidiasis. 
effective regarding faecal and urine samples in an experimental infection model involving rodents and also regarding faecal samples from patients having a confirmed S. stercoralis infection [28]. The present work has evaluated, for the first time, the Strong-LAMP method as a molecular method for detecting $S$. stercoralis in patients' urine samples.

PCR was initially used with F3 and B3 external primers (even though none of the samples became amplified) for verifying whether PCR could be used as a molecular method for analysing urine samples. Single PCR has been used in other studies providing good results for detecting $S$. stercoralis DNA in faecal samples and has been prosed as a complementary molecular method to larval concentration in faecal samples before DNA extraction [42]. PCR F3-B3 (with $0.01 \mathrm{ng}$ Strongyloides spp. DNA detection limit) has been effective in detecting $S$. venezuelensis in faecal samples in an experimental infection model [28].

However, positive amplification results have not been obtained when using PCR F3-B3 on patients' urine samples, meaning that it cannot be proposed as a complementary molecular method for a definitive diagnosis of strongyloidiasis. Nevertheless, using the Strong-LAMP method with samples has led to positive visible amplification results concerning colorimetric change and resolving reaction products on agarose gels.

Eleven of the 24 urine samples analysed had parasitologically positive results for $S$. stercoralis by at least one of the coproparasitological diagnosis methods (i.e., Ritchie technique and/or agar plate culture), and $45.83 \%$ of the samples were positive by Strong-LAMP. Furthermore, 16\% of the samples turned a less pronounced green (indicating amplification) than the rest of the positive samples, and a pattern of bands was obtained which was dimmer on agarose gel. The high protein concentration in these samples could have limited amplification; the 260/280 ratio for absorbance regarding DNA extracted from the samples (often less than 1, most being around 0.5) was well below the expected value for pure nucleic acids (between 1.8 and 2.0 ; $\geq 1.8$ for DNA), thereby limiting analytical purposes. Such high protein concentration would have been the main cause of inhibiting PCR analysing the samples.

Strong-LAMP amplification of two urine samples (I.D: 3 and 4) was obtained in patients having negative faecal sample results by agar plate culture. Given poor sensitivity for coproparasitological technique when analysing a single sample of a patient's faeces, both samples could have proved positive if a serial analysis had been performed (at least three samples from the same patient, taken on alternating days) for visualising S. stercoralis larvae [21].

Regarding serological analysis results, most samples had a serological IVD index greater than $1 \mathrm{OD}$, considered the cut-off limit for considering a result positive by this technique [43]. For example, the sample 3 had a very high IVD index (13.7 OD), indicating anti-S. stercoralis antibodies. It is worth noting that sample 13 which was positive by both agar plate culture and Strong-LAMP had an IVD serological index considered negative (0.005 OD). It has been noted that false negatives sometimes appear when using the IVD serological test [43]. Only one individual who was HIV positive (stage A2) proved positive in all tests carried out in this study. The tests are more efficient for detecting the parasite in such individuals since they may have a hyperinfection syndrome, as observed in a case report by Grossi et al. [44] describing a HIV-positive male having peripheral T-cell lymphoma who developed hyperinfection syndrome and was successfully treated with subcutaneous ivermectin [44]. A study in Brazil by Marchi-Blatt and Aparecida-Cantos [45] compared Strongyloides stercoralis diagnosis techniques on HIV positive and negative patients; it found that HIV positive patients had higher S. stercoralis infection frequency (odds ratio $=5.687$ ) [45]. A study regarding urine analysis in Ethiopia by Hailegebriel et al. [46] demonstrated that agreement between coproparasitological analysis and Strong-LAMP for urine samples in this study was 78\%; it should thus be worthwhile evaluating the test's efficiency in a broader population.

Despite the fact that PCR using external primers F3-B3 and the Strong-LAMP method has been shown to have the same limit for detecting Strongyloides spp. DNA (0.01 ng) [28], the results obtained from analysing the urine samples were consistent with the LAMP method having greater tolerance as described by other authors concerning possible inhibitors in biological samples analysed regarding PCR [47].

Although more studies are needed which would involve increasing the amount of urine samples to be analysed, the present work's results suggest that the Strong-LAMP method and those obtained in faeces [28] have demonstrated that the technique could be used as a complementary molecular tool when diagnosing strongyloidiasis. It could be used as a routine molecular method as it has certain advantages over PCR, i.e., it is cheaper as samples are amplified at constant temperature $\left(59-66^{\circ} \mathrm{C}\right)$ making equipment such as water baths and thermal blocks sufficient, it can detect small concentrations of DNA in samples and is highly specific making it a simple technique to use [48-50].

\section{Conclusions}

The Strong-LAMP molecular diagnosis method was used for the first time for detecting Strongyloides spp. DNA in patients' urine samples. The Strong-LAMP method proved effective in detecting $S$. stercoralis DNA in the urine samples of patients with confirmed strongyloidiasis and/or the serological suspicion of infection by the parasite. The Strong-LAMP method could be used on urine samples as a complementary method for detecting $S$. stercoralis infection.

\section{Data Availability}

All data used to support the findings of this study are included in the manuscript and figures.

\section{Disclosure}

The funders had no role in study design, data collection and analysis, decision to publish, or preparation of the manuscript. 


\section{Conflicts of Interest}

The authors declare that there is no conflict of interest regarding the publication of this paper.

\section{Authors' Contributions}

Pedro Fernández-Soto and Carmen T. Celis-Giraldo equally contributed equally to this work.

\section{Acknowledgments}

We would like to thank Jason Garry for translating the manuscript. This study was supported by the Institute of Health Carlos III, ISCIII, Spain (http://www.isciii.es), grants: RICET RD16/0027/0018, DTS16/00207, PI16/01784, and the European Union cofinancing by FEDER (Fondo Europeo de Desarrollo Regional) "Una manera de hacer Europa". The Universidad del Rosario, Bogotá (Colombia), covered the open access charges.

\section{Supplementary Materials}

Table 1. Individual patients results from the Hospital Clínic de Barcelona, Barcelona, Spain. Table 2. Individual patients results from Hospital de Poniente, El Ejido, Almería, Spain. (Supplementary Materials)

\section{References}

[1] A. Olsen, L. van Lieshout, H. Marti et al., "Strongyloidiasis-the most neglected of the neglected tropical diseases?," Transactions of the Royal Society of Tropical Medicine and Hygiene, vol. 103, no. 10, pp. 967-972, 2009.

[2] R. Toledo, C. Munoz-Antoli, and J. G. Esteban, "Strongyloidiasis with emphasis on human infections and its different clinical forms," Advances in Parasitology, vol. 88, pp. 165-241, 2015.

[3] G. A. Schad, L. M. Aikens, and G. Smith, "Strongyloides stercoralis: is there a canonical migratory route through the host?," The Journal of Parasitology, vol. 75, no. 5, pp. 740-749, 1989.

[4] F. Schär, U. Trostdorf, F. Giardina et al., "Strongyloides stercoralis: global distribution and risk factors," PLoS Neglected Tropical Diseases, vol. 7, no. 7, article e2288, 2013.

[5] T. Thanchomnang, P. M. Intapan, O. Sanpool et al., "First molecular identification of Strongyloides fuelleborni in longtailed macaques in Thailand and Lao People's Democratic Republic reveals considerable genetic diversity," Journal of Helminthology, vol. 93, no. 5, pp. 608-615, 2019.

[6] S. Puthiyakunnon, S. Boddu, Y. Li et al., "Strongyloidiasis-an insight into its global prevalence and management," PLoS Neglected Tropical Diseases, vol. 8, no. 8, article e3018, 2014.

[7] A. Tuyizere, A. Ndayambaje, T. D. Walker et al., "Prevalence of Strongyloides stercoralis infection and other soil-transmitted helminths by cross-sectional survey in a rural community in Gisagara District, Southern Province, Rwanda," Transactions of the Royal Society of Tropical Medicine and Hygiene, vol. 112, no. 3, pp. 97-102, 2018.

[8] M. Dorris and M. Blaxter, "The small subunit ribosomal RNA sequence of Strongyloides stercoralis," International Journal for Parasitology, vol. 30, no. 8, pp. 939-941, 2000.
[9] R. I. Adell and V. D. Márquez, "Estrongiloidiasis: epidemiología, manifestaciones clínicas y diagnóstico. Experiencia en una zona endémica: la comarca de La Safor (Valencia)," Enfermedades Infecciosas y Microbiología Clínica, vol. 25, pp. 38-44, 2007.

[10] P. R. Sánchez, A. P. Guzman, S. M. Guillen et al., "Endemic strongyloidiasis on the Spanish Mediterranean coast," QJM: An International Journal of Medicine, vol. 94, no. 7, pp. 357363, 2001.

[11] G. Ramirez-Olivencia, M. Á. C. Espinosa, A. B. Martín et al., "Imported strongyloidiasis in Spain," International Journal of Infectious Diseases, vol. 18, pp. 32-37, 2014.

[12] W. Winnicki, M. Eder, P. Mazal, F. J. Mayer, G. Sengolge, and L. Wagner, "Prevalence of_Strongyloides stercoralis_infection and hyperinfection syndrome among renal allograft recipients in Central Europe," Scientific Reports, vol. 8, no. 1, article 15406, 2018.

[13] T. B. Nutman, "Human infection with Strongyloides stercoralis and other related Strongyloides species," Parasitology, vol. 144, no. 3, pp. 263-273, 2017.

[14] D. C. Pichard, J. R. Hensley, E. Williams, A. B. Apolo, A. D. Klion, and J. J. DiGiovanna, "Rapid development of migratory, linear, and serpiginous lesions in association with immunosuppression," Journal of the American Academy of Dermatology, vol. 70, no. 6, pp. 1130-1134, 2014.

[15] T. Ali, S. Kaitha, S. Mahmood, A. Ftesi, J. Stone, and M. S. Bronze, "Clinical use of anti-TNF therapy and increased risk of infections," Drug, Healthcare and Patient Safety, vol. 5, pp. 79-99, 2013.

[16] M. F. Khaliq, R. E. Ihle, and J. Perry, "Immunosuppression with antitumour necrosis factor therapy leading to Strongyloides hyperinfection syndrome," Case Reports in Infectious Diseases, vol. 2018, Article ID 6341680, 4 pages, 2018.

[17] D. Buonfrate, F. Formenti, F. Perandin, and Z. Bisoffi, "Novel approaches to the diagnosis of Strongyloides stercoralis infection," Clinical Microbiology and Infection, vol. 21, no. 6, pp. 543-552, 2015.

[18] Y. Suputtamongkol, N. Premasathian, K. Bhumimuang et al., "Efficacy and safety of single and double doses of ivermectin versus 7-day high dose albendazole for chronic strongyloidiasis," PLoS Neglected Tropical Diseases, vol. 5, no. 5, article e1044, 2011.

[19] C. Henriquez-Camacho, E. Gotuzzo, J. Echevarria et al., "Ivermectin versus albendazole or thiabendazole for Strongyloides stercoralis infection," Cochrane Database of Systematic Reviews, article CD007745, 2016.

[20] A. Requena-Mendez, P. Chiodini, Z. Bisoffi, D. Buonfrate, E. Gotuzzo, and J. Munoz, "The laboratory diagnosis and follow up of strongyloidiasis: a systematic review," PLoS Neglected Tropical Diseases, vol. 7, no. 1, article e2002, 2013.

[21] M. Montes, C. Sawhney, and N. Barros, "Strongyloides stercoralis: there but not seen," Current Opinion in Infectious Diseases, vol. 23, no. 5, pp. 500-504, 2010.

[22] F. M. Paula, F. M. Malta, P. D. Marques et al., "Molecular diagnosis of Strongyloides stercoralis among transplant candidates," Transplant Infectious Disease, vol. 20, no. 4, article e12909, 2018.

[23] A. Perera, J. P. Maia, F. Jorge, and D. J. Harris, "Molecular screening of nematodes in lacertid lizards from the Iberian Peninsula and Balearic Islands using 18S rRNA sequences," Journal of Helminthology, vol. 87, no. 2, pp. 189-194, 2013. 
[24] M. R. Watts, G. James, Y. Sultana et al., "A loop-mediated isothermal amplification (LAMP) assay for Strongyloides stercoralis in stool that uses a visual detection method with SYTO-82 fluorescent dye," The American Journal of Tropical Medicine and Hygiene, vol. 90, no. 2, pp. 306-311, 2014.

[25] M. Parida, S. Sannarangaiah, P. K. Dash, P. V. Rao, and K. Morita, "Loop mediated isothermal amplification (LAMP): a new generation of innovative gene amplification technique; perspectives in clinical diagnosis of infectious diseases," Reviews in Medical Virology, vol. 18, no. 6, pp. 407-421, 2008.

[26] K. Dhama, K. Karthik, S. Chakraborty et al., "Loop-mediated isothermal amplification of DNA (LAMP): a new diagnostic tool lights the world of diagnosis of animal and human pathogens: a review," Pakistan Journal of Biological Sciences, vol. 17, no. 2, pp. 151-166, 2014.

[27] Z. K. Njiru, "Loop-mediated isothermal amplification technology: towards point of care diagnostics," PLoS Neglected Tropical Diseases, vol. 6, no. 6, article e1572, 2012.

[28] P. Fernández-Soto, A. Sánchez-Hernández, J. Gandasegui et al., "Strong-LAMP: A LAMP Assay for Strongyloides spp. detection in stool and urine samples. Towards the diagnosis of human Strongyloidiasis starting from a rodent model," PLOS Neglected Tropical Diseases, vol. 10, no. 7, article e0004836, 2016.

[29] P. Fernandez-Soto, V. Velasco Tirado, C. Carranza Rodriguez, J. L. Perez-Arellano, and A. Muro, "Long-term frozen storage of urine samples: a trouble to get PCR results in Schistosoma spp. DNA detection?," PLoS One, vol. 8, no. 4, article e61703, 2013.

[30] A. Petrie and C. Sabin, "Assessing agreement," in Medical Statistics at a Glance, A. Petrie and C. Sabin, Eds., pp. 118-121, Wiley, UK, 3rd edition, 2009.

[31] M. A. Levenhagen and J. M. Costa-Cruz, "Update on immunologic and molecular diagnosis of human strongyloidiasis," Acta Tropica, vol. 135, pp. 33-43, 2014.

[32] D. Buonfrate, M. A. Mena, A. Angheben et al., "Prevalence of strongyloidiasis in Latin America: a systematic review of the literature," Epidemiology and Infection, vol. 143, no. 3, pp. 452-460, 2015.

[33] L. Gétaz, R. Castro, P. Zamora et al., "Epidemiology of Strongyloides stercoralis infection in Bolivian patients at high risk of complications," PLoS Neglected Tropical Diseases, vol. 13, no. 1, article e0007028, 2019.

[34] A. Mir, D. Benahmed, R. Igual et al., "Eosinophil-selective mediators in human strongyloidiasis," Parasite Immunology, vol. 28, no. 8, pp. 397-400, 2006.

[35] C. Carranza-Rodriguez, M. Escamilla-Gonzalez, I. FuentesCorripio, M. J. Perteguer-Prieto, T. Garate-Ormaechea, and J. L. Perez-Arellano, "Helminthosis and eosinophilia in Spain (1990-2015),” Enfermedades Infecciosas y Microbiología Clínica, vol. 36, no. 2, pp. 120-136, 2018.

[36] M. Higashiarakawa, T. Hirata, T. Tanaka et al., "Normal serum IgE levels and eosinophil counts exhibited during Strongyloides stercoralis infection," Parasitology International, vol. 66, no. 1, pp. 807-812, 2017.

[37] V. Luvira, K. Trakulhun, M. Mungthin et al., "Comparative diagnosis of Strongyloidiasis in immunocompromised patients," The American Journal of Tropical Medicine and Hygiene, vol. 95, no. 2, pp. 401-404, 2016.
[38] S. Abdel-Rahman and A. Metwally, "Obstacles in the diagnosis of Strongyloides stercoralis," Parasitologists United Journal, vol. 7, no. 1, pp. 5-12, 2014.

[39] A. Requena-Mendez, D. Buonfrate, Z. Bisoffi, and J. M. Gutiérrez, "Advances in the diagnosis of human Strongyloidiasis," Current Tropical Medicine Reports, vol. 1, no. 4, pp. 207-215, 2014.

[40] N. Lodh, R. Caro, S. Sofer, A. Scott, A. Krolewiecki, and C. Shiff, "Diagnosis of Strongyloides stercoralis: detection of parasite-derived DNA in urine," Acta Tropica, vol. 163, pp. 9-13, 2016.

[41] F. Formenti, G. la Marca, F. Perandin et al., "A diagnostic study comparing conventional and real-time PCR for Strongyloides stercoralis on urine and on faecal samples," Acta Tropica, vol. 190, pp. 284-287, 2019.

[42] H. Moghaddassani, H. Mirhendi, M. Hosseini, M. Rokni, G. Mowlavi, and E. Kia, "Molecular diagnosis of Strongyloides stercoralis infection by PCR detection of specific DNA in human stool samples," Iranian Journal of Parasitology, vol. 6, no. 2, pp. 23-30, 2011.

[43] B. Bon, S. Houze, H. Talabani et al., "Evaluation of a rapid enzyme-linked immunosorbent assay for diagnosis of strongyloidiasis," Journal of Clinical Microbiology, vol. 48, no. 5, pp. 1716-1719, 2010.

[44] P. A. Grossi, D. Lombardi, A. Petrolo et al., "Strongyloides stercoralis hyperinfection in an HIV-infected patient successfully treated with subcutaneous ivermectin," Tropical Medicine and Infectious Disease, vol. 3, no. 2, p. 46, 2018.

[45] J. Marchi Blatt and G. Aparecida Cantos, "Evaluation of techniques for the diagnosis of Strongyloides stercoralis in human immunodeficiency virus (HIV) positive and HIV negative individuals in the city of Itajaí, Brazil," The Brazilian Journal of Infectious Diseases, vol. 7, no. 6, pp. 402-408, 2003.

[46] T. Hailegebriel, B. Petros, and T. Endeshaw, "Evaluation of parasitological methods for the detection of Strongyloides stercoralis among individuals in selected health institutions In Addis Ababa, Ethiopia," Ethiopian Journal of Health Sciences, vol. 27, no. 5, pp. 515-522, 2017.

[47] M. H. Deng, L. Y. Zhong, O. Kamolnetr, Y. Limpanont, and Z. Y. Lv, "Detection of helminths by loop-mediated isothermal amplification assay: a review of updated technology and future outlook," Infectious Diseases of Poverty, vol. 8, no. 1, p. 20, 2019.

[48] P. R. Sahoo, K. Sethy, S. Mohapatra, and D. Panda, "Loop mediated isothermal amplification: an innovative gene amplification technique for animal diseases," Veterinary World, vol. 9, no. 5, pp. 465-469, 2016.

[49] S. Khurana and S. Sethi, "Laboratory diagnosis of soil transmitted helminthiasis," Tropical Parasitology, vol. 7, no. 2, pp. 86-91, 2017.

[50] M. Keikha, "LAMP method as one of the best candidates for replacing with PCR method," Malaysian Journal of Medical Sciences, vol. 25, no. 1, pp. 121-123, 2018. 